Schmerz 2015 $29: 665$

DOI 10.1007/s00482-015-0062-9

Online publiziert: 9. September 2015

(c) Springer-Verlag Berlin Heidelberg 2015

CrossMark

\author{
H. Jochum ${ }^{1}$ - A. Baumgartner-Gruber ${ }^{1}$ S. Brand ${ }^{2,3} \cdot$ H.-F. Zeilhofer ${ }^{1} \cdot$ P. Keel ${ }^{4}$. \\ C.S. Leiggener ${ }^{1}$ \\ ${ }^{1}$ Klinik für Mund-, Kiefer- und Gesichtschirurgie, Universitätsspital, Basel, Schweiz \\ ${ }^{2}$ Zentrum für Affektive, Stress- und Schlafstörungen, Universitäre \\ Psychiatrische Kliniken Basel, Basel, Schweiz \\ ${ }^{3}$ Departement für Sport, Bewegung und Gesundheit, Universität Basel, Basel, Schweiz \\ ${ }^{4}$ Klinik für Psychiatrie und Psychosomatik, Bethesda Spital, Basel, Schweiz
}

\title{
Myofazial nicht gleich myofaszial
}

\section{Erwiderung}

Zum Leserbrief von J.C. Türp (2015) Die korrekte Bezeichnung für Kiefermuskelschmerz. Schmerz DOI 10.1007/s00482-015-0061-x

Originalbeitrag: Jochum $\mathrm{H}$, BaumgartnerGruber A, Brand S, Zeilhofer HF, Keel P,

Leiggener CS (2015) Chronische Kiefer- und Gesichtsschmerzen: Verringerte Schmerzen durch Psychoedukation und Physiotherapie. Schmerz 29:285-292

Herr Prof. Türp präzisiert in seinem Leserbrief die Terminologie von Kiefergelenkschmerzen. Er unterscheidet die Begriffe „myofacial“ (Gesichtsmuskulatur; mimische Muskulatur) und „myofaszial“ (Kiefermuskulatur).

Wir haben im englischen Titel den Begriff „myofacial“ verwendet, im Abstract aber das Beschwerdebild mit „orofacial pain“ beschrieben. Es ist uns in der abschließenden Kontrolle leider nicht aufgefallen, dass unsere Terminologie im Text vom Lektorat zu „myofacial“ geändert worden war.

Eine korrekt verwendete Terminologie zur Bezeichnung von Kiefer- und Gesichtsschmerzen ist insbesondere zum Vergleich von Studien überaus wichtig. Wir danken Herrn Prof. Türp für den wertvollen Hinweis und für seine ausführliche Stellungnahme zur Thematik. Wir stimmen der geäußerten Kritik zu.

\section{Korrespondenzadresse}

\section{PD Dr. Dr. C.S. Leiggener}

Klinik für Mund-, Kiefer- und Gesichtschirurgie Universitätsspital

Spitalstr. 21, 4031 Basel

christoph.leiggener@usb.ch

\section{Einhaltung ethischer Richtlinien}

Interessenkonflikt. H. Jochum, A. BaumgartnerGruber, S. Brand, H.F. Zeilhofer, P. Keel und C.S. Leiggener geben an, dass kein Interessenkonflikt besteht. 


\section{Hier steht eine Anzeige.}

Springer 